Z. Klin. Chem. Klin. Biochem.

11. Jg. 1973, S. $432-434$

\title{
Störung der Eiweißbestimmung im Liquor cerebrospinalis durch myo-Inosit
}

\author{
Von W. Bürgi und H. KaufmanN
}

Zentrallaboratorium, Kantonsspital Aarau, Schweiz

(Eingegangen am 20. März/11. Mai 1973)

In der vorliegenden Arbeit werden die Isolierung und der Nachweis von myo-Inosit als Hexaacetat aus Liquor cerebrospinalis von menschlichen Neugeborenen und Frühgeburten beschrieben. Nach erfolgter Proteinfällung wurde der Uberstand in einer Mischbettsäule entsalzt. Die Abtrennung des myo-Inosit von Glucose erfolgte mittels präparativer Dünnschichtchromatographie.

myo-Inosit kommt in freier Form im menschlichen Liquor cerebrospinalis vor. Er gibt eine positive Biuretreaktion im UV-Bereich und vermag die Eiweißbestimmung im Liquor zu stören, wenn die Liquorproteine nicht gefällt werden.

\section{Interference by myoinositol in the determination of protein in cerebrospinal fluid}

The isolation of myoinositol from cerebrospinal fluid of newborn and premature babies is described. Following protein precipitation, the protein free filtrate was desalted in a Dowex mixed bed column. Separation of myoinositol from glucose was achieved by thin layer chromatography. The hexaacetate derivative of myoinositol isolated from cerebrospinal fluid and of a reference sample were identical with respect to the melting point, retention time in gas-liquid chromatographic analysis and in mass spectroscopy.

Vor einigen Jahren haben wir eine vereinfachte Methode zur Bestimmung von Gesamteiweiß im Liquor cerebrospinalis publiziert. Das Verfahren nützt die höhere Empfindlichkeit der Biuretreaktion im ultravioletten Licht aus. Wie aus Abbildung 1 hervorgeht, steigt die Extinktion des Biuretkomplexes im UV-Bereich mit abnehmender Wellenlänge an und ist bei $334 \mathrm{~nm}$ etwa fünfmal größer als bei $540 \mathrm{~nm}$. Die wichtigsten Merkmale dieser Methode sind in Tabelle 1 zusammengestellt. Die Übereinstimmung mit dem vorherigen Verfahren, bei welchem die Proteine vorgängig der Biuretreaktion durch Ausfällung konzentriert werden, war gut.

Die Erfahrung mit der modifizierten Biuretmethode zeigte, daß diese bei erwachsenen Patienten klinisch zuverlässige Resultate liefert. Hingegen erhielten wir bei Frühgeburten und Neugeborenen fast regelmäßig hohe

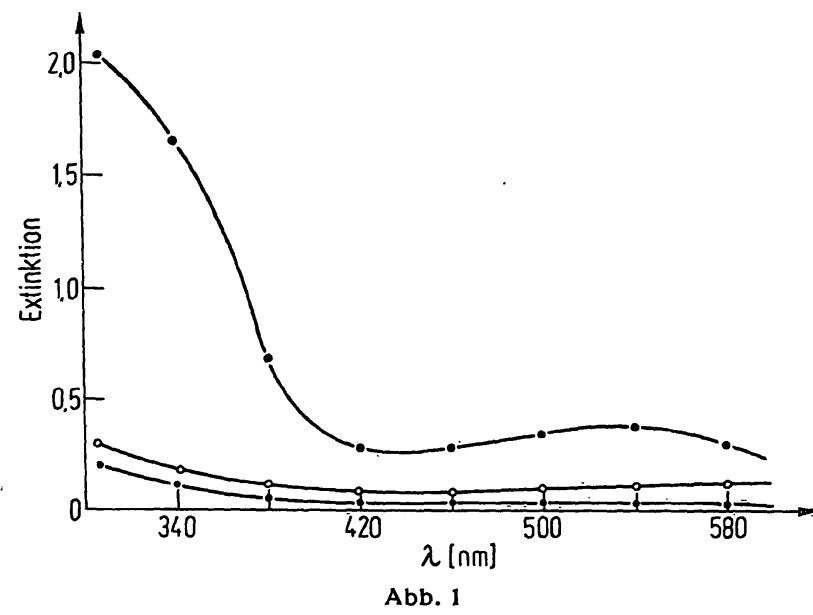

Absorptionsspektrum des Biuretkomplexes von bovinem Serumalbumin (- - $)$, vom Reagenzienleerwert (-O-) und vom Analysenleerwert (- $)$. Die photometrischen Messungen erfolgten gegen dest.
Tab. 1

Merkmale der direkten Liquoreiweißbestimmung

\begin{tabular}{ll}
\hline Prinzip: & Biuret-Reaktion, ohne Fällung der Proteine \\
Reagenz: & modifiziertes Biuret-Reagenz* \\
Probevolumen: & $0,1 \mathrm{ml}$ Liquor \\
& $(0,2 \mathrm{ml}$ bei Mitführen eines Analysenleerwertes) \\
Photometrie: & UV-Bereich, z. B. bei $334 \mathrm{~nm}$ \\
Arbeitsvorschr.: & $0,5 \mathrm{ml}$ Biuret-Reagenz \\
& $0,1 \mathrm{ml}$ Liquar (żentrifugiert) \\
& Photometrie bei 334 $\mathrm{nm}$ \\
& Subtraktion des Reagenzien- und \\
& Analysenleerwertes \\
& Berechnung: mg/l = Extinktion - 4480 \\
*Referenz: & MEHL, J. W., (1945) J. Biol. Chem. 157, 173-180
\end{tabular}

Werte, welche mit den übrigen Befunden nicht übereinstimmten. Ferner konnte immer wieder festgestellt werden, $\mathrm{da} B$ selbst bei sehr hohen Werten die sonst so typische Biuretfarbe in den Testansätzen nicht zu erkennen war. Nachdem grobe Fehler während der Liquorpunktion, beim Transport der Probe und im Laboratorium ausgeschlossen worden waren, wurden Parallelbestimmungen mit einer im Handel erhältlichen Testpackung vorgenommen ${ }^{1}$ ) (nachfolgend als Methode 2 bezeichnet). Dieses Verfahren beruht auf der Konzentrierung der Eiweiße durch Perchlorsäurefällung aus einem Probevolumen von $1 \mathrm{ml}$ vorgängig der Biuretreaktion im Präzipitat. Die Photometrie erfolgt im sichtbaren Bereich zwischen 540 und $550 \mathrm{~nm}$. Die Ergebnisse von 15 Vergleichsuntersuchungen zeigten, daß die Metbode 1, also das direkte Verfahren mit Messung des Biuretkomplexes bei $334 \mathrm{~nm}$, mit Ausnahme eines 47jährigen Patienten und zwei Knaben im Alter von 2 und 10 Jahren bei allen Neugeborenen und Frühgeburten hoch pathologische Werte liefert. Die Resultate

1) Bezugsquelle: Fa. Hoffmann-La Roche, Diagnostica, Basel. 


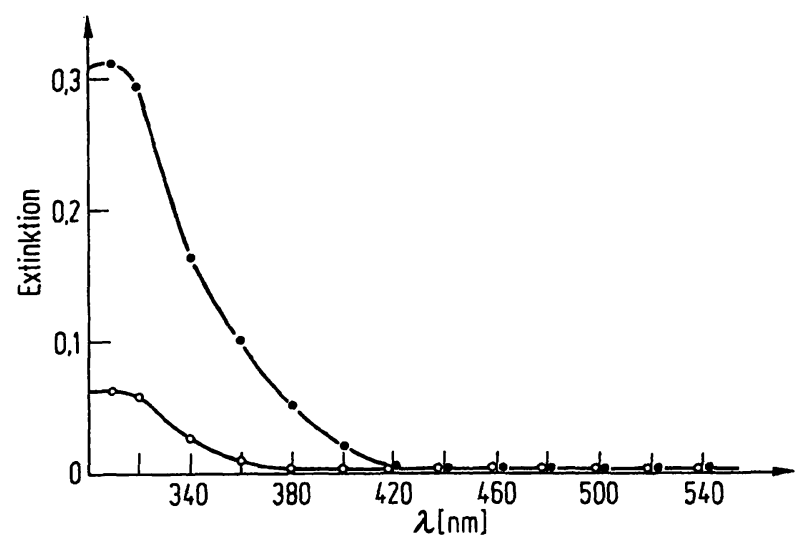

Abb. 2

Absorptionsspektren der Biuretkomplexe von ultrafiltriertem Liquor cerebrospinalis eines Neugeborenen $\left(-0_{-}\right)$und eines 47jährigen Mannes (- O-). Die Analysen wurden nach der Methode 1 vorgenommen; die photometrischen Ablesungen erfolgten gegen den Reagenzienleerwert

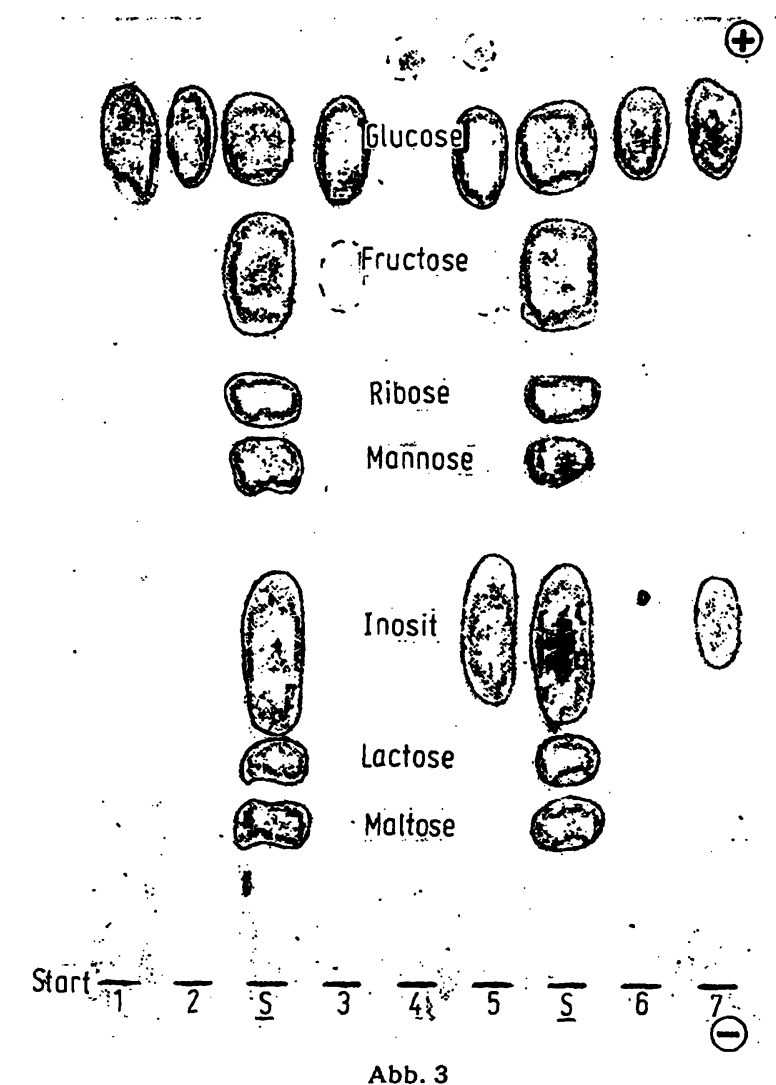

Abb. 3

Hochspannungselektrophorese von unbehandeltem Liquor in $0,05 \mathrm{~mol} / \mathrm{l}$ Na-Tetraboratpuffer, $\mathrm{pH} 9,2$. Die Auftragsmenge betrug $50 \mu \mathrm{l}$. Anfärben mit dem AgNo ${ }_{3}-\mathrm{NaOH}$ Reagenz. Nr. 1, 2, 3, 4, 6: Erwachsene Lactose, myo-Inosit, Mannose, Ribose, Fructose und Glucose in wäßriger Lösung

der Methode 2 hingegen liegen wesentlich niedriger. Sie passen zum klinischen Bild und zu den übrigen Befunden. Zur Abklärung dieser Unstimmigkeiten wurden vorerst zwei Proben in einer Amicon 12 Ultrafiltrierzelle durch Sartorius SM 12136 Membranfilter ultrafiltriert. Von beiden Ultrafiltraten wurden die Biuretreaktion nach Metbode 1 ausgeführt und die Absorptionsspektren aufgenommen. Diese sind in Abbildung 2 aufgezeichnet. Das Ultrafiltrat vom Liquor des 47jährigen Patienten absorbiert im gesamten untersuchten Wellenlängenbereich ultraviolette Strahlen nur gering. Im Gegensatz dazu bildet das Spektrum des Neugeborenenliquors im kurzwelligen Licht einen Buckel, der unterhalb $480 \mathrm{~nm}$ beginnt. Diese Ergebnisse weisen darauf hin, daß im Liquor von Neugeborenen und Frühgeburten eine oder mehrere kleinmolekulare Substanzen vorkommen, die die Eiweißbestimmung stören, indem sie mit dem Biuretreagenz einen Komplex bilden, der die langwelligen ultravioletten Strahlen absorbiert.

Vorversuche zur Isolierung der störenden Substanz Das Ultrafiltrat vom Liquor des Neugeborenen (Abb. 2) wurde säulenchromatographisch entsalzt. Die nachfolgende Dünnschichtchromatographie ergab mit der $\mathrm{AgNO}_{3}-\mathrm{NaOH}-F a ̈ r b u n g$ (2) zwei Flecken, von denen der größere mit dem höheren $\mathrm{R}_{\mathrm{F}}$-Wert als Glucose identifiziert werden konnte. Eine Abtrennung von Glucose aus dem entsalzten Ultrafiltrat mittels Säulenchromatographie an Dowex 2 in der Boratform gelang nicht, da die gesuchte Substanz offensichtlich auch einen Boratkomplex wie die Glucose bildete. Es durfte somit vermutet werden, daß sie eine polyhydroxyartige Struktur aufweist. Tatsächlich konnten in unbehandeltem Liquor mit der Hochspannungselektrophorese in 0,05 mol/1 Na-Tetraboratpuffer, pH 9,2, zwei Flecken nachgewiesen werden, von denen sich der eine als Glucose identifizieren ließ. Der unbekannte Flecken wies die gleiche Wanderungsgeschwindigkeit wie eine Referenzprobe von myo-Inosit auf (Abb. 3).

\section{Isolierung und Identifizierung} von freiem myo-Inosit aus Liquor cerebrospinalis

Das Ausgangsmaterial setzte sich aus Einzelproben von Liquor cerebrospinalis von Frühgeburten und Neugeborenen zusammen. Die Gesamtmenge betrug $18 \mathrm{ml}$. Nach Einstellen des pH auf 4,5 mit $1 \mathrm{~mol} / \mathrm{l}$ Essigsäure wurden unter ständigem Rühren tropfenweise $80 \mathrm{ml}$ absolutes Äthanol zugegeben. Das Proteinpräzipitat wurde abzentrifugiert, der klare Úberstand eingetrocknet und in $15 \mathrm{ml}$ destilliertem Wasser aufgenommen. Die Lipide wurden mit Äther extrahiert und die wäßrige Phase in einer Dowex-Mischbettsäule entsalzt. Das Eluat enthielt neben Glucose eine Substanz, die in der Hochspannungselektrophorese gleich weit wie eine myoInositprobe wanderte. Die Abtrennung der Glucose erfolgte mit präparativer Dünnschichtchromatographie auf Kieselgel F 254-Platten mit $75 \mathrm{ml} \mathrm{n}$-Butanol + $15 \mathrm{ml}$ Pyridin $+10 \mathrm{ml}$ Wasser als Fließmittel. Die myo-InositFraktion, die sich in der nachfolgenden Hochspannungselektrophorese als frei von Glucose erwies, wurde von Kieselgel eluiert und mit einer $1+2$ Mischung von Essigsäureanhydrid und Pyridin acetyliert. Das Acetylierungsprodukt wurde sublimiert und aus Äther umkristallisiert. Die Ausbeute betrug $0,6 \mathrm{mg}$ farbloser Kristalle mit einem Schmelzpunkt von $211^{\circ} \mathrm{C}$. Von einer Referenzprobe myo-Inosit wurde ebenfalls das Hexaacetat hergestellt und dieses auskristallisiert. Der Schmelzpunkt betrug $213^{\circ} \mathrm{C}$. Gaschromatographie mit SE 30 als Trägermaterial und anschließende Massenspektrographie bewiesen die Identität der Referenzsubstanz myo- 


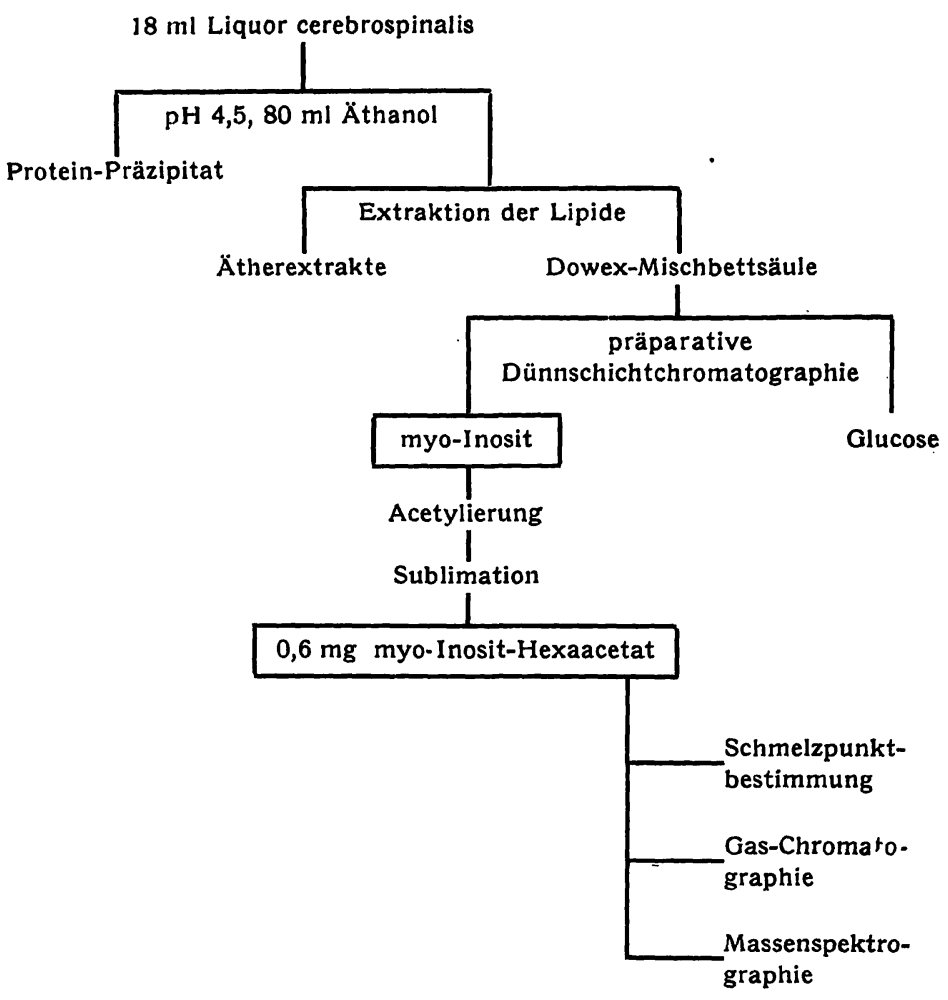
Abb. 4
Schema zur Isolierung von myo-Inosit aus dem Liquor cere- brospinalis von Frühgeburten und Neugeborenen. Einzelheiten siehe Text

Inosit-Hexaacetat mit dem aus dem Liquor isolierten myo-Inosit ${ }^{2}$ ). Die Schritte der Gewinnung von myoInosit sind in Abbildung 4 schematisch dargestellt. myo-Inosit findet sich in freiem Zustand bei Mensch und Tier. In einer ausgedehnten Übersichtsarbeit haben Beckmann und Petith (3) Vorkommen und Konzentration in den Körperflüssigkeiten und Organen von Mensch und verschiedenen Tierarten zusammengestellt. Die Konzentrationen betragen $0,11-0,19 \mathrm{mmol} / 1$ im menschlichen Liquor cerebrospinalis und $0,028-0,06$ $\mathrm{mmol} / \mathrm{l} \mathrm{im}$ Serum. myo-Inosit liegt gebunden in den Phosphatiden, den sog. Inosit-Phosphatiden, vor. Die Bedeutung des freien myo-Inosit ist noch unklar. Erhöhte Konzentrationen im Liquor wurden bei Gehirn- und Rückenmarkstumoren gemessen. Erniedrigte Werte wurden z. B. bei der senilen Gehirn- atrophie, bei der epidemischen Encephalitis und bei der akuten tuberkulösen Meningitis gefunden.

Über die Inositkonzentrationen im Liquor von menschlichen Frühgeburten und Neugeborenen liegen keine Resultate vor. Immerhin darf nicht unerwähnt bleiben, $\mathrm{da} B$ im Foetus der Ziege und des Schafes Werte von $3,06 \mathrm{mmol} / \mathrm{l}$, bzw. 7,8 mmol/l angegeben werden. Die Vermutung liegt somit nahe, daß der myo-Inositgehalt im menschlichen Neugeborenenliquor ebenfalls erhöht ist. Die beim Tier gefundenen Konzentrationen würden die eingangs erwähnten falsch hohen Eiweißresultate jedenfalls erklären.

2) Den Herren Dres. A. LAmpart und W. Vetrer, HoffmánnLa Roche, Basel, sei an dieser Stelle für die Durchführung der gaschromatographischen und massenspektrographischen Analysen gedankt.

\title{
Literatur
}

1. Bürgi, W., Richterich, R. \& Briner, M. (1967), Clin. Chim. Chem. Abstr. 47, 11297-239, 247. - 3. Beckmann, R. \& Petrth, Acta 15, 181-184. - 2. Petronici, C. \& Safina, G. (1953),

M. (1970), Med. Welt 21, 140-146.

\author{
Dr. W. Bürgi \\ Zentrallaboratorium Kantonsspital \\ CH-5001 Aarau
}

